\title{
Chemical zoning of muscovite megacrystal from the Brazilian Pegmatite Province
}

\author{
RÚBIA R. VIANA ${ }^{1}$, HANNA J. EVANGELISTA ${ }^{2}$ and WILLEM B. STERN ${ }^{3}$ \\ ${ }^{1}$ Coordenação do Programa de Pós-Graduação em Geociências, Departamento de Recursos Minerais, ICET \\ Universidade Federal de Mato Grosso, Av. Fernando Correa da Costa, s/n, Coxipó \\ Campus Universitário, 78068-900 Cuiabá, MT, Brasil \\ ${ }^{2}$ Departamento de Geologia, Universidade Federal de Ouro Preto, Morro do Cruzeiro \\ 35400-000 Ouro Preto, Minas Gerais, Brasil \\ ${ }^{3}$ Mineralogisch-Petrographisches Institut, University of Basel, Basel, Switzerland \\ Manuscript received on May 16, 2005; accepted for publication on August 23, 2006; \\ presented by ALCIDES N. SIAL
}

\begin{abstract}
Macroscopically homogenous muscovite plate from the Cruzeiro pegmatite, located in the Eastern Pegmatite Province in Minas Gerais, may show complex distribution patterns of some trace elements. In geochronological and petrological studies, as for example in the distinction of magmatic and post-magmatic mica, the cause of zoning could be taken into consideration. The complex chemical zoning in the studied mica plate can be best explained by growth in an evolving magma followed by alteration due to percolation of hydrothermal fluids. Enrichment of Rb towards the border is interpreted as resulting from the chemical evolution of the residual magma during crystal growth. The depletion in $\left({ }^{\mathrm{IV}} \mathrm{Al}+{ }^{\mathrm{VI}} \mathrm{Al}\right)$ as well as the increase in $(\mathrm{Fe}+\mathrm{Mg})$ and $\mathrm{Si}$ along a fracture could be due to the hydrothermal celadonitic substitution of muscovite. This alteration also caused depletion in the contents of Rb, Ga, Y, Nb, Sn, and Zn and residual concentration of Ti. Elements such as $\mathrm{Ga}, \mathrm{Y}, \mathrm{Nb}, \mathrm{Sn}$, and $\mathrm{Zn}$, rarely considered in the discussion of differentiation or alteration processes in micas, have been shown to be as significant as the alkali-elements.
\end{abstract}

Key words: muscovite, chemical zoning, hydrothermal alteration, Cruzeiro pegmatite.

\section{INTRODUCTION}

Two kinds of zoning were found in micas from pegmatites from the gem-producing Eastern Brazilian Pegmatite Province (EBPP): discontinuous zoning, due to the overgrowth of muscovite on biotite as described by Viana et al. (2003), and chemical zoning of crystals of a single type of mica, investigated in the present paper.

The Cruzeiro pegmatite is located in Governador Valadares region, Minas Gerais State, Brazil (Fig. 1). This pegmatite is composed of three subvertical dikes reaching a thickness of 50 meters. The pegmatite is hosted by quartzite of the Serra da Safira Sequence (Federico et al. 1998). It shows well defined internal

Correspondence to: Profa. Dra. Rúbia Ribeiro Viana E-mail: rubia@cpd.ufmt.br zonation, being composed of quartz, feldspar, muscovite, gem-tourmaline and less commonly beryl (aquamarine variety), garnet, niobotantalate, spodumene, and rarely amblygonite (Bilal et al. 2000, Cassedane et al. 1980).

Muscovite of variable size is common in all pegmatites from the EBPP, mostly as pseudo-hexagonal or fishtail shaped books. The larger crystals are found in the intermediate zone of Cruzeiro Pegmatite, in some cases exceeding $30 \mathrm{~cm}$. A large muscovite plate from this zone was submitted to detailed chemical analysis in order to examine it compositional zoning and to understand the growth patterns of giant crystals. A thin fracture line crosscutting the plate from one border to the other was the only heterogeneity observed.

The chemical variation within the large muscovite plate was investigated by special analytical procedures, 
enabling the detection of trace elements with a higher degree of accuracy than by conventional electron microprobe analysis.

\section{GEOLOGICAL SETTING}

A large quantity and variety of gemstones, particularly aquamarine and tourmaline, is produced in the Eastern Brazilian Pegmatite Province (EBPP), which comprises an area about $800 \mathrm{~km}$ long and $150 \mathrm{~km}$ wide. The pegmatites are spread over eastern Minas Gerais, western Espírito Santo and southern Bahia States (Fig. 1). The EBPP is characterized by a particular geotectonic setting in a Neoproterozoic-Cambrian orogenic belt generated during the Brasiliano-Pan-African cycle, which consisted of a set of orogenies that lasted from about 850 to $550 \mathrm{Ma}$ (e.g., Oliveira et al. 1997, Pinto and PedrosaSoares 2001). The majority of the pegmatites of the EBPP are related to granite intrusions into the Brasiliano mobile belts generated during the consolidation of the Gondwana supercontinent. Biotite and its muscovite overgrowth from the Ipê pegmatite, located near Governador Valadares (Fig. 1), have been dated by the $\mathrm{K} /$ Ar method enabling to establish a crystallization age of $575 \mathrm{Ma}$ as well as a cooling rate of $3.3^{\circ} \mathrm{C}$ for the pegmatite (Viana et al. 2003).

\section{SAMPLING AND EXPERIMENTAL METHODS}

An $80 \times 40 \mathrm{~cm}$ mica plate from the intermediate zone of the Cruzeiro pegmatite was selected for analysis. Mica discs were cut out on 56 points of the muscovite plate. An even, clean crystal surface of $30 \mathrm{~mm}$ diameter was analyzed in the Geochemical Laboratory Institute of Mineralogy and Petrography of Basel University (Switzerland) by means of X-ray Fluorescence Analysis - XFA, (SRS-3400 spectrometer of Siemens-BrukerAXS, Germany, Specplus software) without further preparation using the analytical routine discussed by Stern (2001). No grinding process took place, and hence no contamination (W, Co) induced by sample dressing. No elements were lost in this way due to volatilization $(\mathrm{F}, \mathrm{Cl})$ as is the case with certain conventional preparation techniques such as vitrification (glass beads). The specimen thickness was measured by means of a micrometer and used for thickness correction, essential when high-energy spectral lines are used for analysis.
Since analytical reliability decreases with decreasing concentrations (Fig. 2), elements which are present mostly well above the detection limit were preferably selected for analysis in this study.

The results of the chemical analyses reported in Table I show that the main composition changes little, that is, the chemical variation of the major elements is within the analytical frame of detection. However, some trace elements vary with a factor 2 to 3 , which reflects indeed the changing environment during crystal growth.

\section{PETROLOGICAL CONSIDERATIONS TO ELEMENT DISTRIBUTION PATTERNS}

The locations of the analyzed points and the distribution maps of the compositional contour lines for selected elements are presented in Figure 3. The distribution maps show very similar patterns, although the trends might be the opposite, as for example for Ti and Ga (Fig. 3). The similar distribution of the contour lines is an indication that the chemical zoning is due to the operation of some kind of geological process, i.e., it is not the result of imprecise analytical data that would certainly result in chaotic distribution patterns.

In order to evaluate the element distribution maps within the muscovite plate, the following geological processes are potential causes for the chemical variation in pegmatite minerals:

1 - The chemical evolution of residual magma during the crystallization of the pegmatite magma is responsible for an increase of $\mathrm{Rb}$ and other alkalielements and a decrease of the $\mathrm{K} / \mathrm{Rb}$-ratio during fractionation (e.g. Morteani et al. 1995). Consequently, there is an enrichment of $\mathrm{Rb}$ and depletion of $\mathrm{K} / \mathrm{Rb}$-ratio from center to border of pegmatite minerals such as mica during growth. In melts remaining during fractionated crystallization there is an increase in $\mathrm{Rb}$ and decrease in $\mathrm{Sr}$ and $\mathrm{Ba}$ (Neiva et al. 1987).

2 - Zoning due to hydrothermal alteration and/or growth. In this case it is supposed that the produce enrichment in the celadonitic component that leads to increase $\mathrm{Si}$ and $(\mathrm{Fe}+\mathrm{Mg})$ and diminish $\left({ }^{\mathrm{IV}} \mathrm{Al}+{ }^{\mathrm{VI}} \mathrm{Al}\right)$ when compared to magmatic muscovite (Gomes and Neiva 2000, Demster et al. 1994). 

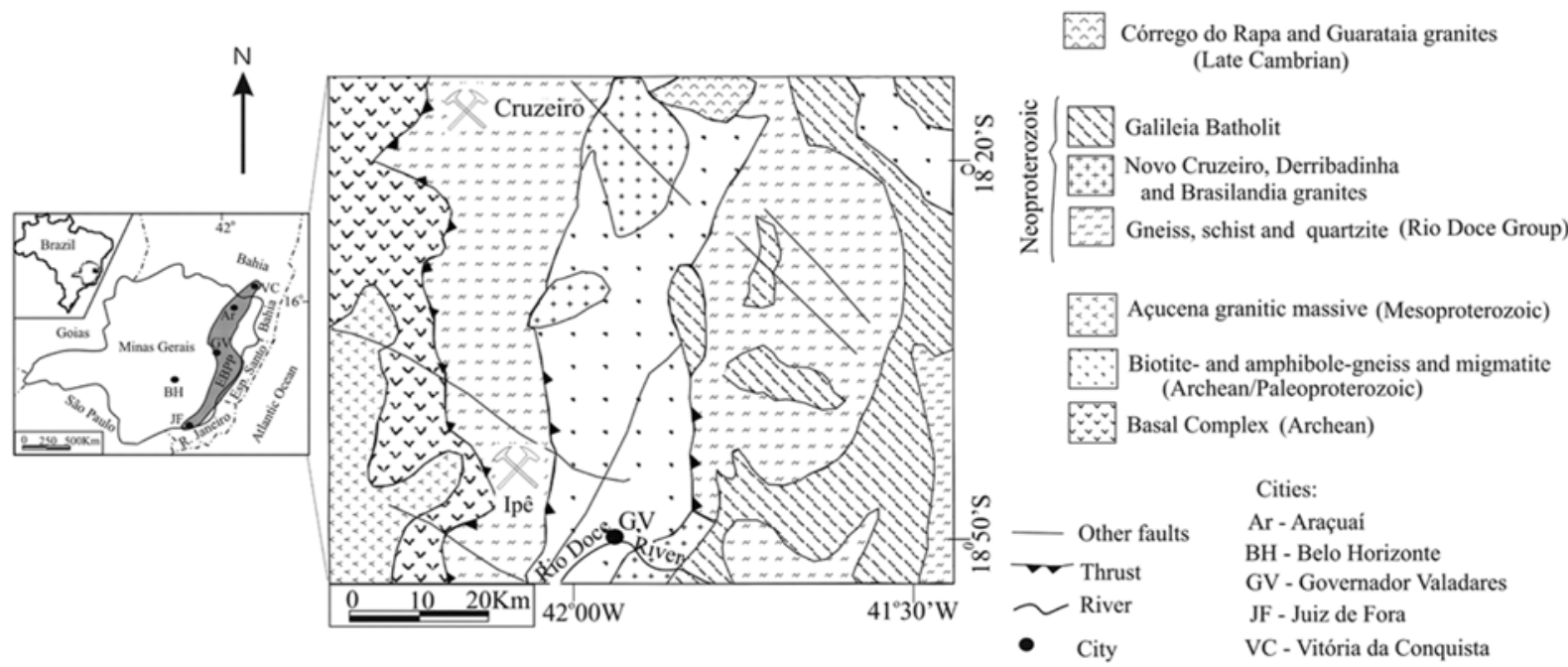

EBPP- Eastern Brazilian Pegmatite Province

Fig. 1 - Simplified geological map of the Araçuaí and Governador Valadares regions with location of the Cruzeiro pegmatite. Outline of the Eastern Brazilian Pegmatite Province (EBPP) is also shown (modified after Oliveira et al. 1997 and Pedrosa-Soares and Wiedemann-Leonardos 2000).

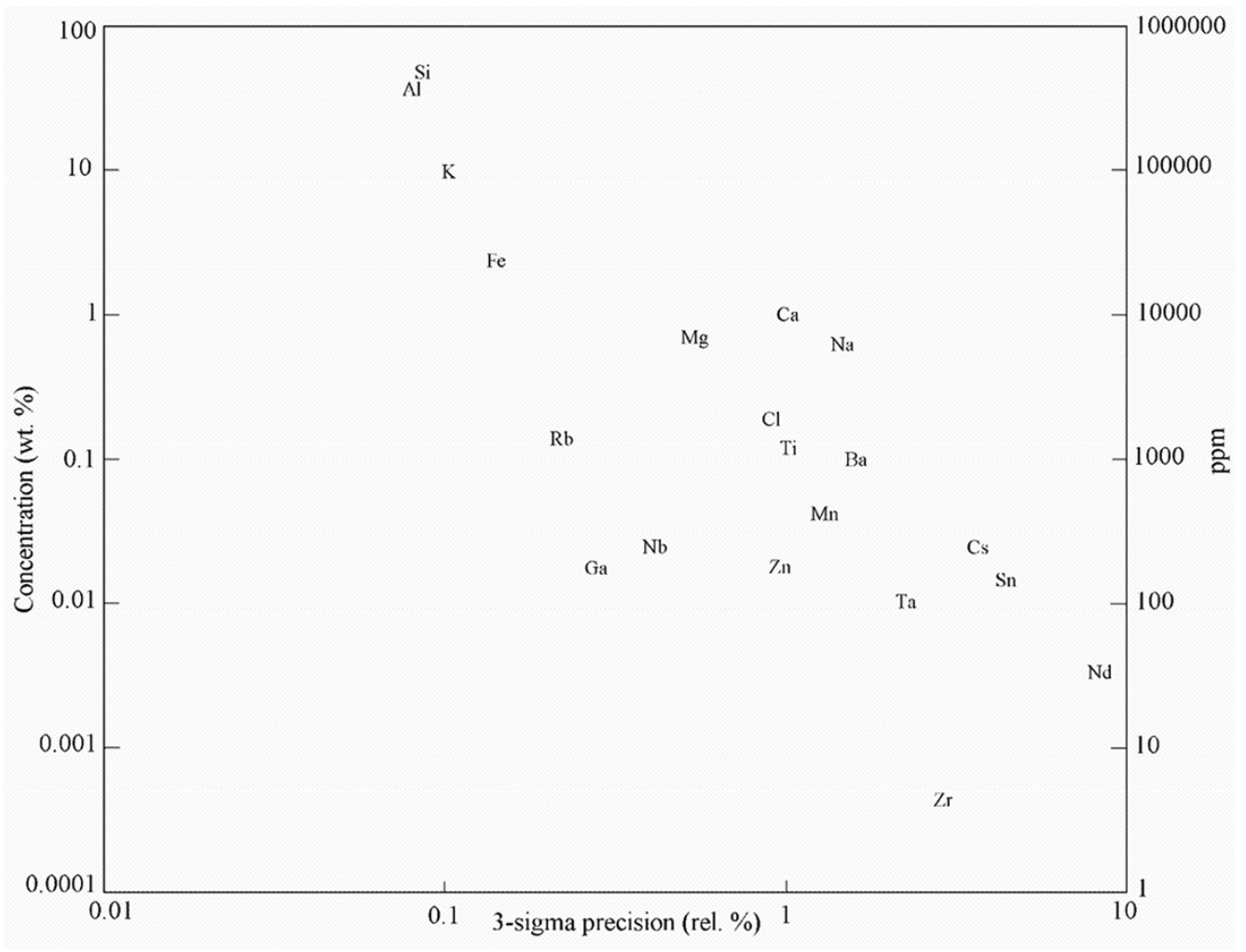

Fig. 2 - Estimated analytical error for analyzed elements at 99\% confidence level. 


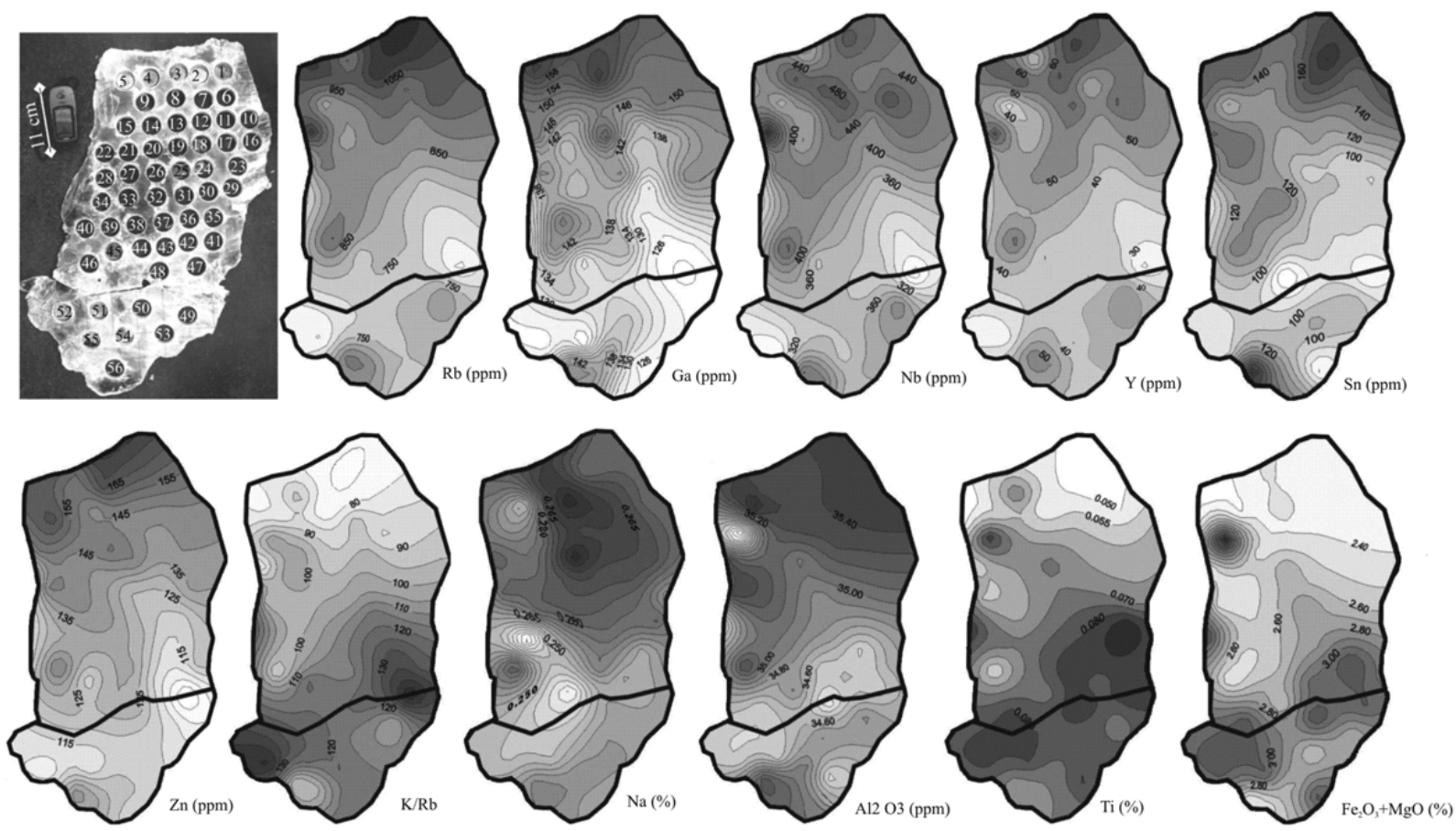

Fig. 3 - Sketch of studied muscovite plate with location of the analyzed points and distribution maps of the compositional contour lines for selected elements.
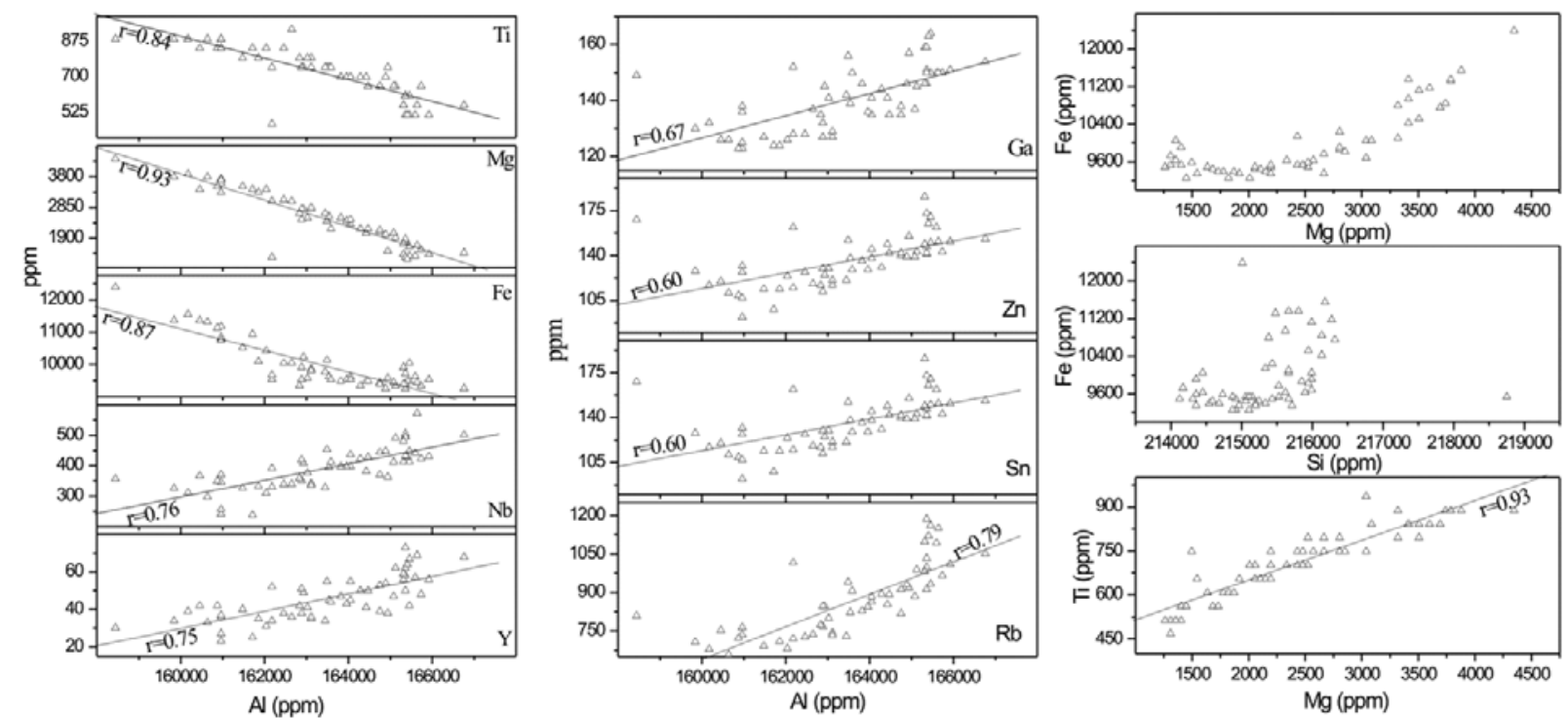

Fig. 4 - Selected bivariant diagrams showing the trend compositional of the muscovite megacrystal studied. Solid line is the best linear fit to the experimental data $(\mathrm{n}=56)$. 
TABLE I

Chemical analyzed of large muscovite from Cruzeiro Pegmatite (oxides in \% Wt and ions in ppm).

\begin{tabular}{|c|c|c|c|c|c|c|c|c|c|c|c|c|c|c|}
\hline & 1 & 2 & 3 & 4 & 5 & 6 & 7 & 8 & 9 & 10 & 11 & 12 & 13 & 14 \\
\hline $\mathrm{SiO}_{2}$ & 45.85 & 45.81 & 45.87 & 46.00 & 45.85 & 45.85 & 45.87 & 45.84 & 45.89 & 45.80 & 46.01 & 45.97 & 45.98 & 46.04 \\
\hline $\mathrm{Al}_{2} \mathrm{O}_{3}$ & 35.36 & 35.37 & 35.42 & 35.49 & 35.45 & 35.28 & 35.39 & 35.39 & 35.37 & 35.38 & 35.67 & 35.37 & 35.32 & 35.31 \\
\hline $\mathrm{Fe}_{2} \mathrm{O}_{3}$ & 2.12 & 2.08 & 2.06 & 2.04 & 2.00 & 2.05 & 2.15 & 2.03 & 2.01 & 2.03 & 1.98 & 1.98 & 2.00 & 2.02 \\
\hline $\mathrm{MnO}$ & 0.05 & 0.05 & 0.04 & 0.04 & 0.04 & 0.04 & 0.05 & 0.04 & 0.04 & 0.05 & 0.04 & 0.04 & 0.04 & 0.03 \\
\hline $\mathrm{MgO}$ & 0.30 & 0.28 & 0.29 & 0.30 & 0.33 & 0.32 & 0.29 & 0.35 & 0.38 & 0.27 & 0.31 & 0.39 & 0.41 & 0.44 \\
\hline $\mathrm{CaO}$ & 0.01 & 0.00 & 0.00 & 0.00 & 0.00 & 0.04 & 0.00 & 0.00 & 0.00 & 0.00 & 0.00 & 0.00 & 0.00 & 0.00 \\
\hline $\mathrm{Na}_{2} \mathrm{O}$ & 0.81 & 0.75 & 0.79 & 0.76 & 0.74 & 0.81 & 0.85 & 0.82 & 0.80 & 0.74 & 0.66 & 0.81 & 0.80 & 0.80 \\
\hline $\mathrm{K}_{2} \mathrm{O}$ & 10.45 & 10.48 & 10.52 & 10.46 & 10.48 & 10.39 & 10.25 & 10.49 & 10.44 & 10.45 & 10.17 & 10.44 & 10.45 & 10.45 \\
\hline $\mathrm{TiO}_{2}$ & 0.12 & 0.11 & 0.11 & 0.11 & 0.14 & 0.16 & 0.11 & 0.13 & 0.13 & 0.11 & 0.12 & 0.13 & 0.14 & 0.14 \\
\hline $\mathrm{P}_{2} \mathrm{O}_{5}$ & 0.01 & 0.02 & 0.01 & 0.01 & 0.01 & 0.02 & 0.02 & 0.01 & 0.01 & 0.01 & 0.02 & 0.01 & 0.01 & 0.01 \\
\hline Sum & 95.08 & 94.95 & 95.11 & 95.21 & 95.04 & 94.96 & 94.98 & 95.10 & 95.07 & 94.84 & 94.98 & 95.14 & 95.15 & 95.24 \\
\hline $\mathrm{H}_{2} \mathrm{O}^{*}$ & 4.5 & 4.5 & 4.5 & 4.5 & 4.5 & 4.5 & 4.5 & 4.5 & 4.5 & 4.5 & 4.5 & 4.5 & 4.5 & 4.5 \\
\hline $\mathrm{F}$ & 0 & 4400 & 0 & 0 & 2800 & 3000 & 2800 & 0 & 2400 & 4000 & 3100 & 0 & 0 & 0 \\
\hline $\mathrm{Cl}$ & 900 & 200 & 1600 & 600 & 500 & 0 & 0 & 1100 & 0 & 200 & 100 & 900 & 400 & 500 \\
\hline $\mathrm{Ba}$ & 920 & 0 & 0 & 0 & 182 & 1182 & 545 & 607 & 263 & 1232 & 524 & 449 & 612 & 0 \\
\hline Cs & 17 & 19 & 7 & 12 & 13 & 19 & 118 & 35 & 16 & 18 & 16 & 13 & 10 & 19 \\
\hline $\mathrm{Nb}$ & 413 & 497 & 442 & 431 & 423 & 363 & 449 & 413 & 506 & 429 & 502 & 494 & 492 & 412 \\
\hline $\mathrm{Rb}$ & 1098 & 1186 & 1094 & 1010 & 966 & 918 & 1161 & 933 & 1033 & 1120 & 1051 & 997 & 988 & 885 \\
\hline $\mathrm{Sn}$ & 149 & 149 & 192 & 151 & 181 & 154 & 132 & 173 & 141 & 157 & 157 & 127 & 149 & 124 \\
\hline $\mathrm{Zn}$ & 186 & 173 & 162 & 151 & 143 & 155 & 170 & 150 & 141 & 165 & 153 & 142 & 143 & 139 \\
\hline $\mathrm{Ga}$ & 159 & 159 & 150 & 151 & 150 & 157 & 164 & 150 & 151 & 163 & 154 & 150 & 145 & 137 \\
\hline $\mathrm{Pb}$ & 38 & 41 & 72 & 34 & 30 & 0 & 0 & 42 & 40 & 25 & 0 & 33 & 35 & 67 \\
\hline $\mathrm{Sb}$ & 0 & 0 & 0 & 0 & 0 & 0 & 0 & 0 & 0 & 0 & 0 & 0 & 0 & 0 \\
\hline $\mathrm{Sr}$ & 0 & 0 & 0 & 0 & 0 & 0 & 0 & 0 & 0 & 0 & 0 & 0 & 0 & 0 \\
\hline $\mathrm{Y}$ & 56 & 73 & 57 & 56 & 48 & 38 & 67 & 42 & 62 & 64 & 68 & 58 & 62 & 47 \\
\hline $\mathrm{K} / \mathrm{Rb}$ & 79 & 73 & 80 & 86 & 90 & 94 & 73 & 93 & 84 & 77 & 80 & 87 & 88 & 98 \\
\hline
\end{tabular}

Hydrothermal muscovite is also supposed to be poorer in $\mathrm{Nb}$ and $\mathrm{Ta}$ (Neiva 1987).

3 - Coalescence of growing crystals by synneusis (Roycroft 1991), which should generate independent zoning in each of the attached crystals surrounded by late stage zones joining the various parts (Shelley 1993). Corrosion followed by later growth of normal euhedral faces is also possible (Roycroft 1989, 1991).

\section{RESULTS AND DISCUSSION}

The following discussion about the chemical variation within the studied mica plate takes into account the above mentioned possible causes of zoning in pegmatite minerals.

Analyzing the selected bivariant diagrams (Fig. 4) it is possible to observe that there are a clear negative correlation between $\mathrm{Ti}, \mathrm{Mg}$ and $\mathrm{Fe}$ and positive correlation between $\mathrm{Nb}, \mathrm{Rb}, \mathrm{Sn}, \mathrm{Zn}, \mathrm{Ga}, \mathrm{Y}$ and Mn, both versus the
Al content. In Figure 4, is also shown a positive correlation of Ti content in function of the $\mathrm{Mg}$ content. On the other hand, the Fe content versus both $\mathrm{Mg}$ and Si present two different trends, negative for lower and positive for higher $\mathrm{Mg}$ and $\mathrm{Si}$ contents. This tendency can be related to presence of $\mathrm{Fe}^{2+}$ and $\mathrm{Fe}^{3+}$ in the samples. No correlation was observed with $\mathrm{K}$ content, meaning that $\mathrm{K} / \mathrm{Rb}$ ratio is controlled only by $\mathrm{Rb}$.

Distribution patterns of indicator elements such as $\mathrm{Rb}$ and $\mathrm{K} / \mathrm{Rb}$ in the studied megacrystal is unrelated to crystal center-border geometry (Fig. 3). Rb shows a gradual increase towards the upper border and a left to right-oriented low crosscutting the lower half of the crystal, which is more or less coincident with the fracture line. Elements showing similar trends to $\mathrm{Rb}$ are $\mathrm{Ga}, \mathrm{Y}, \mathrm{Nb}, \mathrm{Sn}, \mathrm{Zn}, \mathrm{Na}$, and $\left({ }^{\mathrm{IV}} \mathrm{Al}+{ }^{\mathrm{VI}} \mathrm{Al}\right) . \mathrm{K} / \mathrm{Rb}$ ratio, on the other hand, shows an opposite trend, which is also found for $\mathrm{Ti}$ and $(\mathrm{Fe}+\mathrm{Mg})$ and less pronounced also for $\mathrm{Si}$. Other elements, such as $\mathrm{Ba}, \mathrm{K}, \mathrm{Pb}, \mathrm{F}$, not shown in 
TABLE I (continuation)

\begin{tabular}{|c|c|c|c|c|c|c|c|c|c|c|c|c|c|c|}
\hline & 15 & 16 & 17 & 18 & 19 & 20 & 21 & 22 & 23 & 24 & 25 & 26 & 27 & 28 \\
\hline $\mathrm{SiO}_{2}$ & 46.00 & 46.79 & 45.99 & 46.13 & 46.06 & 46.12 & 46.02 & 46.08 & 45.90 & 46.06 & 46.10 & 46.08 & 46.17 & 46.13 \\
\hline $\mathrm{Al}_{2} \mathrm{O}_{3}$ & 35.24 & 34.69 & 33.89 & 35.17 & 34.97 & 35.09 & 35.07 & 35.04 & 35.43 & 35.24 & 35.09 & 34.85 & 34.89 & 34.79 \\
\hline $\mathrm{Fe}_{2} \mathrm{O}_{3}$ & 2.02 & 2.04 & 2.65 & 2.03 & 2.17 & 2.06 & 2.04 & 2.03 & 2.02 & 2.01 & 2.04 & 2.19 & 2.11 & 2.15 \\
\hline $\mathrm{MnO}$ & 0.03 & 0.04 & 0.04 & 0.04 & 0.04 & 0.03 & 0.03 & 0.03 & 0.04 & 0.03 & 0.03 & 0.03 & 0.03 & 0.03 \\
\hline $\mathrm{MgO}$ & 0.45 & 0.28 & 0.93 & 0.44 & 0.52 & 0.50 & 0.52 & 0.54 & 0.36 & 0.46 & 0.53 & 0.60 & 0.60 & 0.65 \\
\hline $\mathrm{CaO}$ & 0.00 & 0.00 & 0.00 & 0.00 & 0.00 & 0.00 & 0.00 & 0.00 & 0.00 & 0.00 & 0.00 & 0.00 & 0.00 & 0.00 \\
\hline $\mathrm{Na}_{2} \mathrm{O}$ & 0.79 & 0.74 & 0.71 & 0.75 & 0.85 & 0.79 & 0.78 & 0.70 & 0.77 & 0.74 & 0.78 & 0.78 & 0.76 & 0.76 \\
\hline $\mathrm{K}_{2} \mathrm{O}$ & 10.44 & 10.28 & 10.38 & 10.48 & 10.31 & 10.51 & 10.45 & 10.52 & 10.47 & 10.45 & 10.50 & 10.44 & 10.47 & 10.44 \\
\hline $\mathrm{TiO}_{2}$ & 0.14 & 0.10 & 0.19 & 0.15 & 0.16 & 0.15 & 0.15 & 0.15 & 0.12 & 0.14 & 0.15 & 0.16 & 0.17 & 0.20 \\
\hline $\mathrm{P}_{2} \mathrm{O}_{5}$ & 0.01 & 0.02 & 0.01 & 0.01 & 0.01 & 0.01 & 0.01 & 0.01 & 0.01 & 0.01 & 0.01 & 0.02 & 0.01 & 0.01 \\
\hline Sum & 95.12 & 94.98 & 94.79 & 95.19 & 95.08 & 95.27 & 95.07 & 95.10 & 95.12 & 95.14 & 95.23 & 95.16 & 95.21 & 95.17 \\
\hline $\mathrm{H}_{2} \mathrm{O}^{*}$ & 4.5 & 4.5 & 4.5 & 4.5 & 4.5 & 4.5 & 4.5 & 4.5 & 4.5 & 4.5 & 4.5 & 4.5 & 4.5 & 4.5 \\
\hline $\mathrm{F}$ & 0 & 3800 & 6700 & 0 & 2600 & 0 & 0 & 2600 & 0 & 2300 & 0 & 0 & 0 & 0 \\
\hline $\mathrm{Cl}$ & 500 & 200 & 1200 & 300 & 200 & 300 & 1400 & 300 & 1000 & 100 & 600 & 600 & 700 & 0 \\
\hline $\mathrm{Ba}$ & 1060 & 254 & 289 & 732 & 201 & 0 & 929 & 0 & 116 & 0 & 0 & 490 & 350 & 1217 \\
\hline $\mathrm{Cs}$ & 11 & 15 & 14 & 15 & 9 & 8 & 12 & 12 & 23 & 10 & 13 & 29 & 14 & 13 \\
\hline $\mathrm{Nb}$ & 445 & 392 & 357 & 382 & 454 & 398 & 398 & 397 & 573 & 371 & 436 & 409 & 342 & 340 \\
\hline $\mathrm{Rb}$ & 912 & 1016 & 809 & 853 & 941 & 864 & 843 & 828 & 1151 & 817 & 883 & 846 & 743 & 736 \\
\hline $\mathrm{Sn}$ & 127 & 162 & 105 & 127 & 114 & 122 & 92 & 89 & 154 & 149 & 116 & 92 & 105 & 92 \\
\hline $\mathrm{Zn}$ & 140 & 162 & 168 & 149 & 152 & 145 & 129 & 136 & 151 & 142 & 138 & 125 & 121 & 118 \\
\hline $\mathrm{Ga}$ & 138 & 152 & 149 & 141 & 156 & 135 & 136 & 146 & 150 & 135 & 141 & 145 & 127 & 137 \\
\hline $\mathrm{Pb}$ & 0 & 55 & 50 & 47 & 39 & 29 & 0 & 49 & 69 & 91 & 46 & 47 & 29 & 0 \\
\hline $\mathrm{Sb}$ & 0 & 0 & 0 & 0 & 0 & 0 & 166 & 186 & 0 & 0 & 0 & 215 & 0 & 214 \\
\hline $\mathrm{Sr}$ & 0 & 0 & 0 & 0 & 0 & 0 & 0 & 0 & 0 & 0 & 0 & 0 & 0 & 0 \\
\hline $\mathrm{Y}$ & 53 & 52 & 30 & 41 & 55 & 45 & 43 & 47 & 69 & 39 & 55 & 49 & 35 & 36 \\
\hline $\mathrm{K} / \mathrm{Rb}$ & 95 & 84 & 106 & 102 & 91 & 101 & 103 & 105 & 76 & 106 & 99 & 102 & 117 & 118 \\
\hline
\end{tabular}

Figure 3, have erratic distribution patterns, while Ta and $\mathrm{Sr}$, which are elements considered of petrological importance, present contents lower than the limit of detection.

Considering the mentioned variation patterns, growth during evolution of residual magma could not be the only cause of zoning, because chemical variation of diagnostic elements such as $\mathrm{Rb}$, which should gradually increase from center to border, shows distribution patterns unrelated to crystal geometric contour. However, the much higher Rb-contents found along the upper border (Fig. 3) suggest that the influence of growth during magmatic differentiation cannot be excluded.

Coalescence of several crystals by synneusis is improbable because the various areas corresponding to the old crystals that should present similar concentric distribution patterns cannot be recognized. The observed lower contents in $\mathrm{Rb}, \mathrm{Ga}, \mathrm{Y}, \mathrm{Nb}, \mathrm{Ta}, \mathrm{Sn}, \mathrm{Zn}, \mathrm{Na}$, and $\left({ }^{\mathrm{IV}} \mathrm{Al}+{ }^{\mathrm{VI}} \mathrm{Al}\right)$ as well as the higher values of $\mathrm{K} / \mathrm{Rb}, \mathrm{Ti}, \mathrm{Si}$, and $(\mathrm{Fe}+\mathrm{Mg})$ along a more or less $\mathrm{E}-\mathrm{W}$ oriented stripe in the southern half of the plate seem to be related to the fracture line (see Fig. 3).

The impoverishment in $\left({ }^{\mathrm{IV}} \mathrm{Al}+{ }^{\mathrm{VI}} \mathrm{Al}\right)$ and the increase in $(\mathrm{Fe}+\mathrm{Mg})$ and $\mathrm{Si}$ near to the fracture region could be due to the celadonitic substitution of muscovite as discussed by Demster (1992) and Gomes and Neiva (2000):

$$
\begin{gathered}
\mathrm{KAl}_{2} \mathrm{Si}_{3} \mathrm{AlO}_{10}(\mathrm{OH})_{2}+\mathrm{Fe}^{3+}+\mathrm{Si}^{4+}= \\
\mathrm{KFe}^{3+}\left(\mathrm{Mg}, \mathrm{Fe}^{2+}\right) \mathrm{Si}_{4} \mathrm{O}^{10}(\mathrm{OH})_{2}+3 A l^{3+} \\
\text { (celadonite) }
\end{gathered}
$$

This substitution is considered to be characteristic of hydrothermal alteration/growth (Demster et al. 1994, Gomes and Neiva 2000). In the studied case alteration could have been caused by hydrothermal fluids circulating along the fracture, implying in a deformational event of late Brasiliano age, following the crystallization of the pegmatite. Elements that were also depleted during the alteration include $\mathrm{Rb}, \mathrm{Ga}, \mathrm{Y}, \mathrm{Nb}, \mathrm{Sn}, \mathrm{Zn}, \mathrm{Na}$. 
TABLE I (continuation)

\begin{tabular}{|c|c|c|c|c|c|c|c|c|c|c|c|c|c|c|}
\hline & 29 & 30 & 31 & 32 & 33 & 34 & 35 & 36 & 37 & 38 & 39 & 40 & 41 & 42 \\
\hline $\mathrm{SiO}_{2}$ & 46.12 & 46.03 & 45.97 & 46.18 & 46.20 & 46.09 & 46.23 & 46.24 & 45.96 & 45.93 & 46.07 & 46.19 & 46.23 & 46.13 \\
\hline $\mathrm{Al}_{2} \mathrm{O}_{3}$ & 34.59 & 35.37 & 35.18 & 34.98 & 34.75 & 34.36 & 34.66 & 34.26 & 34.99 & 34.87 & 34.43 & 34.54 & 34.43 & 34.62 \\
\hline $\mathrm{Fe}_{2} \mathrm{O}_{3}$ & 2.34 & 2.01 & 2.03 & 2.06 & 2.15 & 2.42 & 2.23 & 2.47 & 2.04 & 2.05 & 2.31 & 2.25 & 2.32 & 2.16 \\
\hline $\mathrm{MnO}$ & 0.03 & 0.04 & 0.04 & 0.03 & 0.03 & 0.03 & 0.03 & 0.03 & 0.05 & 0.03 & 0.03 & 0.03 & 0.03 & 0.03 \\
\hline $\mathrm{MgO}$ & 0.73 & 0.40 & 0.47 & 0.55 & 0.66 & 0.81 & 0.73 & 0.83 & 0.47 & 0.54 & 0.71 & 0.75 & 0.80 & 0.71 \\
\hline $\mathrm{CaO}$ & 0.00 & 0.00 & 0.00 & 0.00 & 0.00 & 0.00 & 0.00 & 0.00 & 0.00 & 0.00 & 0.00 & 0.00 & 0.00 & 0.00 \\
\hline $\mathrm{Na}_{2} \mathrm{O}$ & 0.69 & 0.76 & 0.76 & 0.76 & 0.75 & 0.68 & 0.72 & 0.68 & 0.57 & 0.68 & 0.73 & 0.67 & 0.69 & 0.68 \\
\hline $\mathrm{K}_{2} \mathrm{O}$ & 10.56 & 10.46 & 10.48 & 10.49 & 10.50 & 10.57 & 10.51 & 10.38 & 10.68 & 10.62 & 10.40 & 10.57 & 10.56 & 10.47 \\
\hline $\mathrm{TiO}_{2}$ & 0.18 & 0.13 & 0.14 & 0.16 & 0.18 & 0.19 & 0.18 & 0.19 & 0.16 & 0.17 & 0.19 & 0.17 & 0.19 & 0.17 \\
\hline $\mathrm{P}_{2} \mathrm{O}_{5}$ & 0.02 & 0.01 & 0.01 & 0.01 & 0.01 & 0.01 & 0.01 & 0.01 & 0.01 & 0.01 & 0.01 & 0.01 & 0.01 & 0.01 \\
\hline Sum & 95.26 & 95.21 & 95.08 & 95.23 & 95.24 & 95.16 & 95.30 & 95.09 & 94.92 & 94.90 & 94.89 & 95.19 & 95.26 & 94.99 \\
\hline $\mathrm{H}_{2} \mathrm{O}^{*}$ & 4.5 & 4.5 & 4.5 & 4.5 & 4.5 & 4.5 & 4.5 & 4.5 & 4.5 & 4.5 & 4.5 & 4.5 & 4.5 & 4.5 \\
\hline $\mathrm{F}$ & 0 & 0 & 2500 & 0 & 0 & 0 & 0 & 2900 & 5600 & 4400 & 4100 & 0 & 0 & 3400 \\
\hline $\mathrm{Cl}$ & 700 & 600 & 500 & 100 & 300 & 0 & 100 & 100 & 0 & 0 & 0 & 200 & 0 & 300 \\
\hline $\mathrm{Ba}$ & 426 & 110 & 0 & 243 & 346 & 1599 & 0 & 481 & 0 & 927 & 1672 & 832 & 670 & 738 \\
\hline $\mathrm{Cs}$ & 1 & 13 & 11 & 116 & 13 & 10 & 12 & 10 & 92 & 61 & 12 & 24 & 19 & 13 \\
\hline $\mathrm{Nb}$ & 238 & 432 & 428 & 395 & 339 & 297 & 310 & 311 & 412 & 377 & 345 & 327 & 241 & 333 \\
\hline $\mathrm{Rb}$ & 590 & 912 & 892 & 822 & 726 & 658 & 681 & 678 & 906 & 800 & 763 & 691 & 577 & 709 \\
\hline $\mathrm{Sn}$ & 78 & 138 & 127 & 141 & 89 & 92 & 87 & 78 & 130 & 111 & 100 & 84 & 68 & 81 \\
\hline $\mathrm{Zn}$ & 98 & 147 & 142 & 138 & 127 & 111 & 124 & 117 & 129 & 130 & 132 & 114 & 92 & 114 \\
\hline $\mathrm{Ga}$ & 124 & 146 & 135 & 139 & 128 & 126 & 126 & 132 & 150 & 141 & 138 & 127 & 123 & 124 \\
\hline $\mathrm{Pb}$ & 61 & 40 & 50 & 55 & 46 & 34 & 62 & 33 & 32 & 0 & 44 & 40 & 38 & 33 \\
\hline $\mathrm{Sb}$ & 0 & 0 & 0 & 0 & 216 & 0 & 183 & 187 & 0 & 0 & 0 & 216 & 208 & 194 \\
\hline $\mathrm{Sr}$ & 16 & 0 & 0 & 0 & 14 & 0 & 0 & 18 & 33 & 28 & 0 & 0 & 0 & 0 \\
\hline$Y$ & 25 & 50 & 50 & 45 & 38 & 33 & 31 & 39 & 44 & 41 & 36 & 40 & 23 & 35 \\
\hline $\mathrm{K} / \mathrm{Rb}$ & 149 & 95 & 98 & 106 & 120 & 133 & 128 & 127 & 98 & 110 & 113 & 127 & 152 & 123 \\
\hline
\end{tabular}

Ti-contents, on the other hand, may have been increased due to (i) residual concentration because of the immobile character of $\mathrm{Ti}$ or (ii) reaction/alteration of other phases, e.g. rutile needles included in muscovite.

The similarity of the trends of $\mathrm{Ga}, \mathrm{Y}, \mathrm{Nb}, \mathrm{Sn}$, and $\mathrm{Zn}$ to the trend of $\mathrm{Rb}$, which is considered as being an important petrological indicator, demonstrates that in case of the studied mica these elements show the same geochemical behavior as Rb. Therefore elements such as Ga, that shows significant variation, can also be valuable indicators for discrimination of different mica generations in rocks.

\section{ACKNOWLEDGMENTS}

This work was partially supported by Coordenação de Aperfeiçoamento de Pessoal de Nível Superior (CAPES), Conselho Nacional de Desenvolvimento Científico e Tecnológico (CNPq) and Fundação de Amparo a Pesquisas do Estado de Minas Gerais (FAPEMIG)
(Brazil) and by the Intra-muros funding by Geochemical Laboratories/Basel University.

\section{RESUMO}

Um grande cristal de muscovita, macroscopicamente homogêneo, procedente do Pegmatito Cruzeiro, localizado na Província Pegmatítica Oriental, em Minas Gerais, exibe padrão de distribuição complexa para alguns elementos traços. Em estudos geocronológicos e petrológicos, como, por exemplo, na separação entre micas magmáticas e pós-magmáticas, a causa de zoneamento deve ser levada em consideração. O complexo zoneamento químico no cristal de mica estudado é melhor explicado pelo crescimento em um magma evoluído, seguido pela alteração, proveniente da percolação de fluidos hidrotermais. $\mathrm{O}$ enriquecimento de $\mathrm{Rb}$ nas bordas é interpretado como resultado da evolução química do magma residual durante o crescimento do cristal. A diminuição em $\left({ }^{\mathrm{IV}} \mathrm{Al}+{ }^{\mathrm{VI}} \mathrm{Al}\right)$, bem como o aumento de $(\mathrm{Fe}+\mathrm{Mg})$ e $\mathrm{Si}$ ao longo da fratura é explicado pela substituição hidrotermal celadonítica da muscovita. A altera- 
TABLE I (continuation)

\begin{tabular}{|c|c|c|c|c|c|c|c|c|c|c|c|c|c|c|}
\hline & 43 & 44 & 45 & 46 & 47 & 48 & 49 & 50 & 51 & 52 & 53 & 54 & 55 & 56 \\
\hline $\mathrm{SiO}_{2}$ & 45.92 & 46.20 & 46.01 & 46.16 & 46.20 & 46.26 & 46.14 & 46.20 & 46.10 & 46.13 & 46.27 & 45.96 & 46.03 & 46.19 \\
\hline $\mathrm{Al}_{2} \mathrm{O}_{3}$ & 35.36 & 34.69 & 34.84 & 34.19 & 34.84 & 34.43 & 34.83 & 34.41 & 34.96 & 34.32 & 34.43 & 35.27 & 35.14 & 34.89 \\
\hline $\mathrm{Fe}_{2} \mathrm{O}_{3}$ & 2.01 & 2.07 & 2.04 & 2.43 & 2.12 & 2.39 & 2.00 & 2.38 & 2.09 & 2.43 & 2.30 & 1.98 & 2.00 & 2.10 \\
\hline $\mathrm{MnO}$ & 0.04 & 0.03 & 0.04 & 0.03 & 0.03 & 0.03 & 0.03 & 0.03 & 0.03 & 0.03 & 0.03 & 0.04 & 0.04 & 0.03 \\
\hline $\mathrm{MgO}$ & 0.37 & 0.65 & 0.53 & 0.81 & 0.60 & 0.77 & 0.57 & 0.75 & 0.57 & 0.73 & 0.79 & 0.43 & 0.47 & 0.61 \\
\hline $\mathrm{CaO}$ & 0.00 & 0.00 & 0.00 & 0.00 & 0.00 & 0.00 & 0.00 & 0.00 & 0.00 & 0.00 & 0.00 & 0.00 & 0.00 & 0.00 \\
\hline $\mathrm{Na}_{2} \mathrm{O}$ & 0.78 & 0.65 & 0.60 & 0.69 & 0.69 & 0.65 & 0.70 & 0.66 & 0.71 & 0.70 & 0.66 & 0.72 & 0.72 & 0.69 \\
\hline $\mathrm{K}_{2} \mathrm{O}$ & 10.41 & 10.59 & 10.78 & 10.41 & 10.59 & 10.50 & 10.50 & 10.59 & 10.61 & 10.66 & 10.49 & 10.47 & 10.51 & 10.54 \\
\hline $\mathrm{TiO}_{2}$ & 0.12 & 0.16 & 0.16 & 0.19 & 0.16 & 0.18 & 0.17 & 0.18 & 0.16 & 0.18 & 0.18 & 0.15 & 0.15 & 0.16 \\
\hline $\mathrm{P}_{2} \mathrm{O}_{5}$ & 0.01 & 0.01 & 0.01 & 0.01 & 0.01 & 0.01 & 0.01 & 0.02 & 0.01 & 0.01 & 0.01 & 0.01 & 0.01 & 0.01 \\
\hline Sum & 95.02 & 95.05 & 95.01 & 94.92 & 95.24 & 95.22 & 94.96 & 95.22 & 95.24 & 95.20 & 95.16 & 95.02 & 95.06 & 95.22 \\
\hline $\mathrm{H}_{2} \mathrm{O}^{*}$ & 4.5 & 4.5 & 4.5 & 4.5 & 4.5 & 4.5 & 4.5 & 4.5 & 4.5 & 4.5 & 4.5 & 4.5 & 4.5 & 4.5 \\
\hline $\mathrm{F}$ & 3200 & 3600 & 4700 & 4800 & 0 & 0 & 4800 & 0 & 0 & 0 & 0 & 2900 & 2800 & 0 \\
\hline $\mathrm{Cl}$ & 600 & 0 & 0 & 100 & 500 & 0 & 0 & 400 & 100 & 1300 & 0 & 0 & 0 & 700 \\
\hline $\mathrm{Ba}$ & 0 & 350 & 0 & 634 & 0 & 531 & 535 & 378 & 585 & 0 & 1478 & 344 & 457 & 237 \\
\hline Cs & 12 & 76 & 1 & 77 & 8 & 11 & 10 & 31 & 12 & 8 & 1 & 41 & 13 & 11 \\
\hline $\mathrm{Nb}$ & 481 & 330 & 352 & 326 & 419 & 371 & 358 & 350 & 328 & 367 & 257 & 449 & 423 & 336 \\
\hline $\mathrm{Rb}$ & 980 & 720 & 767 & 707 & 846 & 735 & 773 & 722 & 730 & 752 & 591 & 931 & 894 & 733 \\
\hline $\mathrm{Sn}$ & 138 & 114 & 54 & 89 & 111 & 97 & 116 & 114 & 103 & 65 & 81 & 197 & 143 & 122 \\
\hline $\mathrm{Zn}$ & 149 & 115 & 130 & 128 & 112 & 127 & 117 & 109 & 121 & 120 & 107 & 139 & 131 & 117 \\
\hline $\mathrm{Ga}$ & 146 & 128 & 132 & 130 & 127 & 136 & 135 & 123 & 142 & 126 & 125 & 146 & 144 & 129 \\
\hline $\mathrm{Pb}$ & 40 & 0 & 0 & 41 & 58 & 0 & 28 & 34 & 41 & 39 & 27 & 0 & 0 & 37 \\
\hline $\mathrm{Sb}$ & 0 & 0 & 240 & 224 & 0 & 0 & 0 & 0 & 0 & 143 & 182 & 0 & 0 & 0 \\
\hline $\mathrm{Sr}$ & 0 & 0 & 0 & 0 & 0 & 0 & 0 & 0 & 0 & 0 & 0 & 0 & 0 & 0 \\
\hline $\mathrm{Y}$ & 59 & 34 & 38 & 34 & 51 & 37 & 42 & 42 & 34 & 42 & 27 & 54 & 50 & 36 \\
\hline $\mathrm{K} / \mathrm{Rb}$ & 88 & 122 & 117 & 122 & 104 & 119 & 113 & 122 & 121 & 118 & 147 & 93 & 98 & 119 \\
\hline
\end{tabular}

* estimated value.

ção hidrotermal causou, também, a diminuição nos conteúdos de $\mathrm{Rb}, \mathrm{Ga}, \mathrm{Y}, \mathrm{Nb}$, Sn e Zn ao longo desta fratura, além da concentração residual de Ti. Elementos tais como, Ga, Y, Nb, Sn, e Zn, pouco considerados em discussão de diferenciação ou processos de alteração, mostraram significância tanto quanto os elementos alcalinos.

Palavras-chave: muscovita, zoneamento químico, alteração hidrotermal, pegmatito Cruzeiro.

\section{REFERENCES}

Bilal E, Correia-Neves JM, FuZiKaWA K, Horn AH, Marciano VRPRo, Fernandes MLS, Moutte J, Mello FM ANd NASRaoui M. 2000. Pegmatites in southeastern Brazil. RBG 30: 234-237

Cassedane JP, Cassedane JO And SAuer DA. 1980. The Cruzeiro mine past and present. Mineral Rec 11: 363-370.
DEMSTER TJ. 1992. Zoning and recrystallization of pengitic micas: implications for metamorphic equilibration. Contr Min Petr 109: 526-537.

Demster TJ, Tanner PWG and Ainsworth P. 1994. Chemical zoning of white micas. Am Mineral 79: 536544.

Federico M, ANdreozzi GB, Luchesi S, GraZiani G AND CÉSAR-Mendes J. 1998. Compositional variation of tourmaline in the granitic pegmatite dykes of the Cruzeiro mine, Minas Gerais, Brazil. Can Mineral 36: 415-431.

Gomes MEP AND NeIVA AMR. 2000. Chemical zoning of muscovite from the Ervedosa granite, northern Portugal. Mineral Mag 64: 347-358.

Morteani G, Preinfalk C, Spieger W and Bonalumi A. 1995. The Achala granitic complex and the pegmatites of the Sierras Pampeanas (northwest Argentina): A study of differentiation. Econ Geol 90: 636-647. 
NEIVA AMR. 1987. Geochemistry of white micas from Portuguese tin and tungsten deposits. Chem Geol 63: 299317.

NeIVA AMR, NeIVA JMC AND PARRY SJ. 1987. Geochemistry of the granitic rocks and their minerals from Serra da Estrela, Central Portugal. Geo Cosmo Acta 51: 439-454.

Oliveira MJR, FÉboli WL And Pinto CP. 1997. Geologia estrutural e tectônica. In: Projeto Leste. Província Pegmatítica Oriental. Programa de Levantamento Geológico Básico do Brasil, CPRM. Belo Horizonte, MG, Brasil, p. 98-119.

Pedrosa-SoARes AC AND Wiedeman-Leonardos CM. 2000. Evolution of the Araçuaí Belt and its connection to the Ribeira Belt, Eastern Brazil. In: TECTONic Evolution of South AmericA, $31^{\text {st }}$ International Geologic Congress, Rio de Janeiro, RJ, Brazil, p. 265-285.

Pinto CP And Pedros A-SoARes AC. 2001. Brazilian gem provinces. Aus Gem 21: 2-16.
ROYCROFT P. 1989. Zoned muscovite from the Leinster Granite, SE Ireland. Mineral Mag 53: 663-665.

ROYCROFT P. 1991. Magmatically zoned muscovite from the peraluminous two-mica granites of the Leinster batholith, southeast Ireland. Geology 19: 437-440.

SHELley D. 1993. Igneous and Metamorphic Rocks under the Microscope. Chapman \& Hall, London, 445 p.

STERN WB. 2001. XRF-Analysis of Geological Standard Materials: Performance of "Standardless" Measuring Routines for Major and Minor Components. ICP Inf Newsl 27: 196-200.

Viana RR, MantÄri I, Henjes-Kunst F And JoRdtEvangelista H. 2003. Age of pegmatites from eastern Brazil and implications of mica intergrowths on cooling rates and age calculations. J South Am Ear Sci 16: 493501. 